\title{
Technological effect of processing with the organic granular media flows
}

\author{
Yevgeniy Y. Krupenya, Marina A. Boyko*, Antonina P. Shishkina \\ Technological institute, Don State Technical University, 346780 Azov, Russian Federation
}

\begin{abstract}
There has been carried out the analysis of approaches how to model vibrational processing by granulated media. The vibroprocessing model has been developed, that provides effective parts' surfaces finishing work with granules of stone organic media initially based on the approach of energy impact of the granule flow on the processed surface. Specific volumetric metal scrap has been used as the main characteristic of processing with organic media its physical aim is increase rate of thickness of the material removed from the surface in the frames of definite velocity and pressure of the media.It is shown that metal scrap depends on media flow velocity, granular media height of the loading column, conditions that form stationary circulation motion of the media. Taking into account the analysis of experimental studies results of amplitude-frequency characteristics influence on the metal scrap during of abrasive granules vibroprocessing, there has been disclosed some certain dependence of the specific volume metal scrap while organic media processing, considering threshold amplitude and frequency of oscillations of the working tank that made it possible to observe the effect of surface treatment is.The established dependence describing the effective vibroprocessing conditions with stone organic media that was achieved due to experimental data which allows to assume validity of the obtained model.
\end{abstract}

\section{Introduction}

Organic media are an independent group of processing media, their usage and application in the manufacturing machine spare parts technology expand the technological range possibilities of vibration processing and increase the efficiency. However, insufficient research of these media, the absence of techniques for finishing works operations projects with their appliance can minimize the area of their technological implication. Therefore the aim of the given research is to justify consistency of vibroprocessing which provides effective finishing works of the parts surfaces with granules of stone organic media.

\section{Processing modeling with pellet flows of stone organic media}

The article [3] represents the experimental studies of the connection between movement parameters of the abrasive medium in vibro containers and metal scrap intensity, the article

\footnotetext{
* Corresponding author: boyko.tecnology@gmail.com
} 
indicates the following consistency. Metal surfaces streamlined with medium at a high velocity, in case of other equal conditions a greater metal removal velocity is demonstrated. In case of constant velocity of the medium flow, the scrap is always higher with increasing "pressure» of the medium itself due to its greater height above the sample or external created by the loading device. In such experiments material, shape, size and orientation of the samples within the flow were deliberately retained unchanged to distinguish the genuine parameters contribution of moving abrasive medium to the dynamics of the metal scrap.

As the main characteristic of the processing with organic media, specific volumetric metal scrap $\gamma \mathrm{sp}$ is used with the dimension mph, its physical meaning is the increase rate of the thickness of the material removed from the surface at a given velocity and pressure of the medium. As the main factors of the process: medium density $-\rho(\mathrm{kg} / \mathrm{m} 3)$; additional external and internal «hydrostatic pressure» of the medium - p $(\mathrm{Pa})$; the average flow velocity of the medium is $\mathrm{v}(\mathrm{mph})$.

In accordance with the adopted concept of the removal description let's consider organic granular medium to be complete and use the hydrodynamic analogy.

The density of energy flow is one of the characteristics of moving complete media, which is amount of energy «flowing out» per time unit from volume unit resolved in the medium [4]:

$$
E=\rho \cdot v \cdot\left(\frac{v^{2}}{2}+\varepsilon\right)+p \cdot v,
$$

where $\rho$ - density; $v=|v|^{2}$ - velocity; $\mathrm{p}$ - pressure и $\varepsilon$ - internal energy per medium volume unit.

Neglecting thermal effects in granular medium [5], it's possible to obtain an expression for the energy density flow in the formula:

$$
E=\left(\frac{\rho \cdot v^{2}}{2}+p\right) \cdot v
$$

The first term is the kinetic energy transferred per time unit passing through the surface unit with medium mass, the second one is the work forcing the medium carried out by pressure. In such a way, formula (2) characterizes both the kinematics and the energy of medium flow and cutting flow characteristic only in case when the cutting granules characteristics and the orientation of the processed surface are fixed.

In case of fixed flow direction relatively to the surface the formula for the metal scrap can be written down in a scalar form:

$$
\gamma_{\text {уд }}=E \cdot \Pi_{\varphi}
$$

where $\varphi$ index demonstrates mutual orientation of the surface and the flow.

Taking into account the dimension of the energy flow: $\mathrm{E}(\mathrm{kg} / \mathrm{h} 3)$ and the volume metal scraper, the dimension of $\mathrm{P} \varphi$ should be: $\mathrm{m} \cdot \mathrm{h} 2 / \mathrm{kg}$. Thus, the inverse of $\mathrm{P} \varphi: \Sigma(\mathrm{kg} / \mathrm{m}$. h2) has the dimension of mechanical voltage. Then the formula for the metal scrap should be the following:

$$
\gamma_{\text {уд }}=\frac{E}{\Sigma_{\phi}}=\frac{\left(\rho \cdot v^{2} / 2+p\right) \cdot v}{\Sigma_{\phi}},
$$

it means that the volumetric specific metal scrap is proportional to the energy density flow of the organic granular medium. Whereas the parameters $\Sigma$ and $\mathrm{P}$ have the meaning of the 
«resistance module» and the fracture compliance parameter correspondently characterized of the given material surface exposed to the flow of the given granular medium. Correspondence (4) gives qualified description of the established consistency that increase of speed and «pressure» independently increases the specific metal scrap in case of medium intermittence the metal scrap stops.

In order to use this correspondence in practice, the velocity of the medium in the working tank and $\Sigma$ parameter should be known.

The carried out researches in works $[6,10]$ have given the written evidence that metal scrap depends on the medium flow velocity, the height of the loading column of the granular medium. The conditions for the formation of a stationary circulatory motion of the medium along the slightly eccentric (close to the circle) ellipses are:

$$
\frac{H}{L} \cong 1 ; \frac{H}{d_{\text {ч }}}>6 ; \frac{(A \cdot \omega)^{2}}{g \cdot H}<0,5,
$$

where $H$ - loading medium height; $L$ - tank section length; $d_{p}$ - medium particles auxiliary diameter.

$\Sigma$ index understood as abrasive deterioration module depends purely on the abrasive nature and the material characteristic, which is considered to be certain complex parameter characterizing micromechanics of abrasive deterioration in the given tribosystem [7]. $\Sigma$ module is constant and independent of the dynamic medium state, it can be expressed as a function of the physico-mechanical characteristic of the processed material.

Assuming the working tank is not too elongated or flattered along the axis, it is possible to express the loading height $\mathrm{H}$ through the working volume $v$ of the tank. Then the formula that determines the hydrostatic pressure created in the working tank is the following:

$$
p=\rho \cdot g \cdot H=\rho \cdot g \cdot \sqrt[3]{v}
$$

In order to define medium flow velocity [8,9] it should be noted that in case of stabilized flow movement along the vibrating surface, flow velocity is proportional to vibro velocity of the surface itself. According to [7] max velocity of the circulating medium movement inside U-shape tank is determined with the correspondence:

$$
v_{\max }=\frac{A \cdot \omega}{2}=\frac{A \cdot 2 \pi \cdot f}{2}=A \cdot f,
$$

its validity is proved by the independent experimental results [6].

Fig. 1 represents the results of flow velocity measuring in case of amplitude variations and tank vibration frequency.

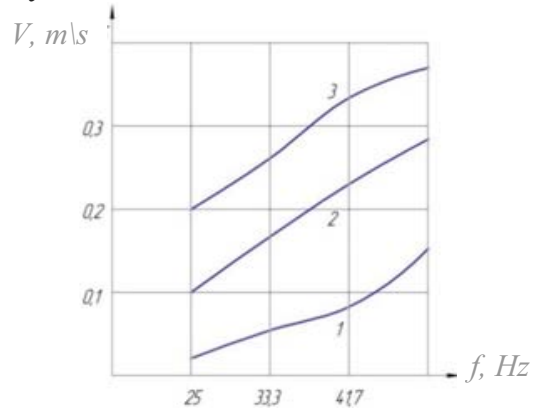

Fig. 1. Dependence of the medium flow circulation velocity on the amplitude and tank vibration frequency [6]: 1) $\mathrm{A}=0.5 \mathrm{~mm}$;) $\mathrm{A}=1.5 \mathrm{~mm}$; 3) $\mathrm{A}=2.5 \mathrm{~mm}$ 
Placing (6) and (7) into (4), the achieved result is:

$$
\gamma_{\text {уд }}=\frac{\rho \cdot \mathrm{A} \cdot \mathrm{f}}{\Sigma} \cdot\left(\frac{\mathrm{A}^{2} \cdot \mathrm{f}^{2}}{2}+\pi \cdot \mathrm{g} \cdot \sqrt[3]{\mathrm{v}}\right)
$$

In the acquired formula $\rho, \Sigma$ characterizes qualities of the processing medium, whereas $A, f, \sqrt[3]{v}$ - dynamic regime and tank size.

However based on the experimental data analysis presented in $[3,6]$, significant drawback of formula (5) should be noted that is the absence of a threshold amplitude and a threshold frequency of oscillations as below them there is no granular organic medium movement and naturally no material scrap.

The work [11] represents the dependence of metal scrap on the amplitude of oscillations on a machine with U-shaped tank of 25 liters capacity, loaded by $2 / 3$. The experiments were carried out without the usage of process liquid. Crushed crumb was used as abrasive medium washed with soda ash. The oscillation frequency was $25 \mathrm{~Hz}$. Characteristic curve was created based on the research results shown in fig. 2 which clearly demonstrates the scrap growth with increasing oscillations amplitude.

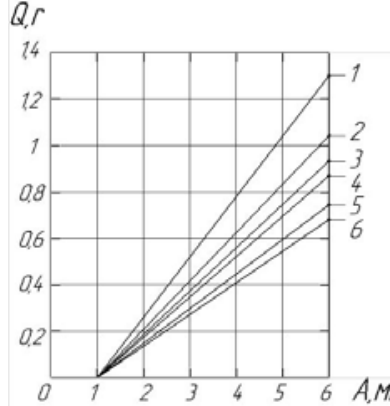

a)

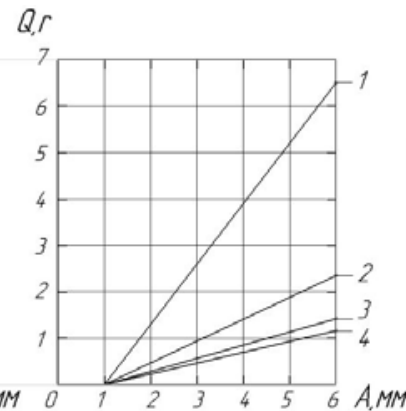

b)

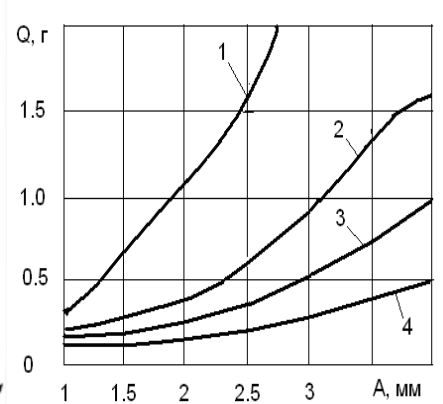

c)

Fig. 2 - Dependence of mass metal scrap from the samples on the oscillations amplitude (abrasive EB63STK granulation $25-40 \mathrm{~mm}$, frequency $25 \mathrm{~Hz}$, time 3 hour)

a)1-SCH-12-28; 2-Steel3; 3-PF-KCH; 4- steel 45; 5-Steel 6 (tempering);

6- U10A(tempering)[10]; b) 1- Babbit B-83; 2- Bronze Br 014; 3- D1; 4-A13 [11];

c) 1- Babbit B-83; 2- bronze Br014; 3-Cast iron C412; 4- steel y8 (tempering) [6]

As it follows from fig. 2 only amplitude less than $1 \mathrm{~mm}$ can be such threshold amplitude. The analysis of sufficient number of experimental data demonstrates the threshold amplitude depends on the vibration frequency. Whilst with frequency increase, the threshold amplitude decreases. The explanation of such phenomenon is represented in work [12], etc. $[1,2,3,6]$, devoted to the dynamics of granular materials subjected to vibration. The analysis of the equations of particles dynamics located on the rough vibrating surface shows that the motion in the «tossing» mode (this is the very mode realized in the vibratory) can begin with the magnitude of the vibration overload [13]:

$$
\Gamma=A \cdot \omega^{2} / g>1
$$

where $g=9,8 \mathrm{mps}^{2}$ - free fall acceleration

Returning to the results of fig. 2 when the experiment was conducted at frequency of 25 $\mathrm{Hz}$, the threshold amplitude will be: 


$$
A_{0} \cdot \omega^{2} \geq g \Rightarrow A_{0} \cong \frac{g}{4 \pi \cdot f^{2}} ; A_{0}>0,4 \mathrm{~mm}
$$

As it follows from [9] the figure $A_{0}$ depends on the particles friction over the supporting surface, the particles layer thickness and their density however in the absence of condensing factors, $A_{0}$ can be defined by the formula with a sufficient accuracy (error up to $15 \%)(10)$.

Given above researches allow to write down the dependence of the specific volume scrap (8) considering threshold amplitude:

$$
\gamma_{\text {уд }}=\frac{\rho\left(A-A_{0}\right) \cdot f}{\Sigma} \cdot\left(\frac{\left(A-A_{0}\right)^{2} \cdot f^{2}}{2}+\pi \mathrm{g} \sqrt[3]{v}\right), A>A_{0}
$$

The dependence curves in Fig. 3 demonstrate that at low frequencies without medium motion, the metal scrap is not observed. In case of frequency increase above the optimal one, corresponding to the most intensive loading movement, scrap increase is significantly lower.

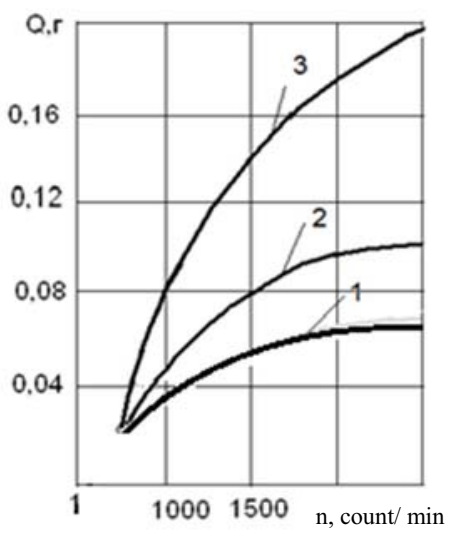

Fig. 3. Dependence of mass metal scrap from the sample on oscillation frequency (abrasive EB63STK granulation 25-40mm, amplitude 1,5 mm, time 1 hour): 1- Bronze Br014; 2- Al3; 3- Steel St3 [6].

Therefore, introduction of threshold frequency similar to the amplitude appears to be proper, beginning with that abrasive medium can come into motion. Obviously this threshold frequency $f_{0}$ depends on the amplitude of the excited oscillations, however the experimental data of the works $[1,6,11]$ show that figure $f_{0}$ is within the range of $10-15 \mathrm{~Hz}$ for the majority of vibration machines with circulation motion character.

Thus the model of the specific volume scrap including the amplitude and frequency dependences will be the following:

$$
\gamma_{\mathrm{yд}}=\frac{\rho \cdot\left(A-A_{0}\right) \cdot\left(f-f_{0}\right)}{\Sigma} \cdot\left(\frac{\left(A-A_{0}\right)^{2} \cdot\left(f-f_{0}\right)^{2}}{2}+\pi \cdot g \cdot \sqrt[3]{v}\right) ; A>A_{0}, f>f_{0},
$$

Expressing $\Sigma$ module as a function of the physico-mechanical characteristics of the processed material and in particular its hardness $\Sigma=\mathrm{K} \cdot \mathrm{HV}$ where $\mathrm{K}$ is experimentally established complex coefficient characterizing physico-mechanical and biological properties of the organic medium granules, also processing conditions (with the usage of 
process liquid or without one), a set of dependencies is received which describes the vibration processing with organic granular media consisting of crushed fruit tree stones:

$$
\begin{gathered}
g_{y d}=\frac{r \times\left(A-A_{0}\right) \times\left(f-f_{0}\right)}{K \times H V} \times\left(\frac{\left(A-A_{0}\right)^{2} \times\left(f-f_{0}\right)^{2}}{2}\right)+p \times g \times \sqrt[3]{v} \\
A>A_{0}, f>f_{0} \\
A_{0}=\pi \cdot \frac{g}{4 \cdot \pi^{2} \cdot f_{0}^{2}} ; \\
\frac{H}{L} \cong 1 ; \frac{H}{d_{\mathrm{u}}}>6 ; \frac{(A \cdot \omega)^{2}}{g \cdot H}<0,5
\end{gathered}
$$

\section{Conclusion}

The dependence of metal scrap and the conditions received within the modeling process are presented in the form of inequalities which correspond to the stationary circulating motion of organic granular medium along elliptical trajectories, which provide efficiency of parts surface vibro processing with stone organic media. Unless these conditions are considered the dynamic state of the process will be different from normal, whilst the formula for the metal scrap can be incorrect.

\section{References}

1. A.P. Babichev, I.A. Babichev, Principles of the vibro technology (Publishing Center DSTU, Rostov-on-Don, 2008)

2. M.A. Tamarkin, Technological optimisation background to process spare parts with free abrasives: published summary of dissertation: $\mathrm{PhD}$ of tech. science (Rostov-on-Don, 1995)

3. S.N. Shevtsov, Comp. dinamic modell. of gran. media in vibrot. mach (Publishing house SKNTS VS, Rostov-on-Don, 2001)

4. S.F. Yatsun, E. V. Zhuravleva, Reports journal IV scientific conference (Kursk, 1999)

5. M. Jaeger, S. R. Nagel, R. P. Behringer, Rev. Mod. Phvs, 68, 1259 (1996)

6. E.Y. Krupenya Efficiency icrease of details processing based on organic nature media: published summary of dissertation ... PhD of tech. science (Rostov-on-Don. 2011).

7. S. N. Coppersmith [et al.] Phys. Rev. E., 53, 5, 4673 (1996)

8. C. E. Bremen, S. Ghosh, C. R. Wassgren, J. Appl. Mech., 63, 1 (1996)

9. R. L. Brown, J. C. Richards, Principles of powder mechanics (Oxford, 1970)

10. F. A. Zenz, D. F. Othmer, Fluidization and fluid-particle systems (New York, 1960).

11. E. A. Dyachenkko, Efficiency icrease of details processing baed on ecologic chюracteristics: published summary of dissertation ... PhD of tech. science (Rostovon-Don, 2004)

12. I. I. Blekhman, E. E. Lavendel, I. F. Goncharevitch, Vibration in engineering (1979)

13. N. C. Mehta, J. M. Smith., E. W. Comings, Industrial Engineering Chemistry (1957) 